\title{
Associações do abandono paterno e fatores socioeconômicos sobre o desenvolvimento
}

\author{
da linguagem \\ Paternal abandonment associations and socioeconomic factors on language development \\ Asociaciones de abandono paterno y factores socioeconómicos en el desarrollo del lenguaje
}

Recebido: 16/08/2021 | Revisado: 26/08/2021 | Aceito: 09/09/2021 | Publicado: 11/09/2021

Gabriela da Silva Leandro

ORCID: https://orcid.org/0000-0001-8188-3383 Universidade do Estado do Pará, Brasil E-mail: gabrielaleandro48@gmail.com

Adriele Francisca da Silva Souza

ORCID: https://orcid.org/0000-0003-2511-2490 Universidade do Estado do Pará, Brasil E-mail: adriele.fsouza@ aluno.uepa.br

Gabriela de Souza Lima

ORCID: https://orcid.org/0000-0003-4832-9780 Universidade do Estado do Pará, Brasil E-mail: gabriela.lima@ aluno.uepa.br

Maria Nery da Silva de Oliveira

ORCID: https://orcid.org/0000-0001-7556-7552 Universidade do Estado do Pará, Brasil E-mail: nerymaria715@gmail.com

Luma Nicole Gomes de Oliveira Lopes

ORCID: https://orcid.org/0000-0003-1234-249X Universidade do Estado do Pará, Brasil E-mail: lumanicole90@gmail.com

Daniella do Socorro Vilacorta da Cruz ORCID: https://orcid.org/0000-0003-0732-3163 Universidade do Estado do Pará, Brasil E-mail: daniellacruz56v@gmail.com

Elson Ferreira Costa

ORCID: https://orcid.org/0000-0003-4115-9029 Universidade Federal do Pará, Brasil E-mail: elsonfcosta@gmail.com Ivete Furtado Ribeiro Caldas

ORCID: https://orcid.org/0000-0002-2095-101X Universidade do Estado do Pará, Brasil E-mail: ivbeiro@yahoo.com.br

\begin{abstract}
Resumo
O desenvolvimento da linguagem depende da interação de fatores biológicos, ambientais e psicossociais existentes nos diversos contextos em que as crianças estão inseridas. Objetivo: Avaliar a associação do abandono paterno e fatores socioeconômicos sobre o desenvolvimento da linguagem de crianças em uma creche pública no município de Marabá (PA). Método: Trata-se de um estudo de caráter transversal, realizado com 62 crianças entre 3 a 6 anos. Os intrumentos utilizados foram o Instrumento de Medição do Nível de Pobreza e o Teste de Triagem de Desenvolvimento de Denver II. Para análise estatística foram utilizados os testes Qui-quadrado, Teste $G$ e o Teste U, todos com nível de significância de 5\%. Resultados: Quanto ao nível de pobreza, $55(88.70 \%)$ crianças apresentavam renda baixa superior e $13(20.97 \%)$ crianças apresentaram suspeita de atraso na linguagem. Vinte e cinco (89.30\%) crianças, cujos pais trabalhavam por tarefa, apresentaram desfecho questionável. E, 11 (39.30\%) crianças que foram abandonadas totalmente pelo pai apresentaram resultado questionável na linguagem. Conclusão: Variáveis sociais como abandono paterno e fatores socioeconômicos possuem influência direta sobre o desenvolvimento da linguagem infantil.
\end{abstract}

Palavras-chave: Crianças; Desenvolvimento da linguagem; Fatores sociais; Abandono infantil.

\begin{abstract}
Language development depends on the interaction of biological, environmental and psychosocial factors existing in the different contexts in which children are inserted. Objective: To evaluate the association of paternal abandonment and socioeconomic factors on the language development of children in a public day care center in the city of Marabá (PA). Method: This is a study of character transverse, carried out with 62 children from 3 to 6 years old. The
\end{abstract}


instruments used were the Poverty Level Measurement Instrument and the Denver II Developmental Screening Test. Statistical analysis the ram used are the tests are Qui-quadrado, Test $G$ and Test U, all with a significance level of 5\%. Results: As for the level of poverty, 55 (88.70\%) children had a higher low income and $13(20.97 \%)$ children had suspected language delay. Twenty-five $(89.30 \%)$ children, whose parents worked per job, had questionable outcome. And, 11 (39.30\%) children who have been abandoned entirely by s father s had questionable results in the language. Conclusion: Social variables such as paternal abandonment and socioeconomic factors have a direct influence on child language development.

Keywords: Children; Language development; Social factors; Child abandonment.

\section{Resumen}

El desarrollo del lenguaje depende de la interacción de factores biológicos, ambientáis e psicossociais existentes. Objetivo: Evaluar la asociación del abandono paterno y los valores socioeconómicos en el desarrollo del lenguaje de los niños en una guardería pública del municipio de Marabá (PA). Método: Se trata de un estudio transversal, realizado con 62 niños de entre 3 y 6 años. Los instrumentos utilizados fueron el Instrumento de Medición del Nivel de Pobreza y el Prueba de detección de Denver Development II. Para el análisis estadístico se utilizaron las pruebas de Qui-quadrado, Prueba $G$ y Prueba U, todos con un nivel de signifiancia del 5\%. Resultados: En cuanto al nivel de pobreza, $55(88,70 \%)$ los niños tenían de bajos ingresos más alto y 13 (20,97\%) niños tenían sospecha de retraso del lenguaje. Veinticinco $(89,30 \%)$ niños cuyos padres trabajaron por tarea, presentó un resultado cuestionable. Y 11 $(39,30 \%)$ niños que fueron totalmente abandonados por su padre presentó resultado cuestionable en el lenguaje. Conclusión: Variables sociales como el abandono paterno y los factores socioeconómicos tener una influencia directa en el desarrollo del lenguaje de los niños.

Palabras clave: Niños; Desarrollo del lenguaje; Factores sociales; Abandono infantil.

\section{Introdução}

O desenvolvimento infantil é caracterizado como um processo de mudanças contínuas e dinâmicas nos variados campos do comportamento humano, como a aquisição de novas habilidades motoras, cognitivas, linguísticas e psicossociais que ocorrem na infância (Zago et al., 2017). Os anos iniciais de vida da criança são essenciais para o desenvolvimento neuropsicomotor (DNPM), período em que estão especialmente suscetíveis a sofrer influência de fatores bioecológicos. Tratase, portanto, de um processo complexo em que há interação de fatores biológicos individuais, relacionados à herança genética; e fatores externos como ambiente físico, cultural, emocional e social, nos quais o infante está inserido (Lima, Cavalcante, \& Costa, 2016; Flores, García-Gomez, \& Zunzunegui, 2014).

A linguagem é um campo do desenvolvimento infantil de destaque, que compreende a utilização sistemática de símbolos linguísticos com o objetivo de comunicação. É um instrumento social usado para transmissão, classificação, combinação e resumo de informações entre os indivíduos; primordial para diversos processos da vida humana, como socialização, aprendizado e integração a sua cultura (Costa, Cavalcante, \& Dell'Aglio, 2015; Mélo, Lucchesi, Junior, \& Signorelli, 2020).

Os fatores externos que influenciam no desenvolvimento linguístico são os relacionados ao ambiente no qual a criança vive, destacando-se o nível socioeconômico. Esta é uma variável complexa, resultante de fatores como renda familiar, escolaridade do cuidador, ocupação dos pais/familiares, que influenciam diretamente nas características do ambiente doméstico, nas interações entre pais e filhos, rotina familiar e na oferta de estímulos que afetam o desenvolvimento infantil no domicílio (Morais, Carvalho, \& Magalhães, 2016; Jacobsen, Moraes, Wagner, \& Trentini, 2013).

Estudos apontam que níveis socioeconômicos mais elevados, principalmente quanto a escolaridade dos cuidadores, estão relacionados a um melhor desfecho intelectual das crianças. Ademais, estudos mostram que cuidados sob médio ou elevado nível socioeconômico tendem a aprimorar o desenvolvimento das habilidades linguísticas durante a infância. Dessa maneira, pais com maior nível de escolaridade ao usarem vocabulário diversificado com os filhos, podem levar ao desenvolvimento mais eficiente da linguagem, melhores hábitos de leitura e capacidade de escrita (Aarnoudse-Moens et al., 2013; Catale et al., 2012; Filippetti, 2011; Khanam \& Nghiem, 2016). 
Nesse contexto socioeconômico, a pobreza familiar é uma variável importante no desenvolvimento da linguagem na infância e está relacionada a diversos co-fatores que podem se tornar fatores de risco, como família com grande número de membros, pequena renda familiar, menor escolaridade, habitação precária e acesso escasso a serviços de saúde. É um fenômeno multifacetado que extrapola questões econômicas e incluem aspectos políticos, sociais e culturais (Tran, Luchters, \& Fisher, 2017). Crianças expostas a condições de pobreza por um grande período estão propensas a atrasos no desenvolvimento com maior frequência e severidade (Araújo, Melo, \& Israel, 2017; Richter et al., 2017).

Outro aspecto a ser considerado é o papel da figura paterna, tendo em vista que a relação entre pai e criança propicia um aspecto positivo para o desenvolvimento infantil (Anme et al., 2012). A paternidade é demonstrada como um importante preditor do desenvolvimento infantil, que se dá mediante a vários fatores: harmonia familiar, facilitando maior afetividade entre os membros da família; melhor dinâmica da família, pela divisão dos afazeres domésticos; participação no cuidado da criança, como na alimentação e higiene, incrementando a proximidade entre pai e filho e também participação em brincadeiras, que aumentam interação entre pai e filho. A ausência do pai age como fator de risco no desenvolvimento infantil, por menor interação e relacionamento com a criança, menor rede de suporte à mãe e apoio nas condições socioeconômicas da família (Manfroi, Macarini, \& Vieira, 2011; Trapp \& Andrade, 2017).

Dessa forma, a família possui papel preponderante em todos as fases do desenvolvimento infantil. É através dela que a criança encontrará um ambiente facilitador para o desenvolvimento de suas potencialidades. Crianças que crescem em ambiente familiar com relações sadias entre os pais, recebem maior suporte emocional que proporcionam melhor desenvolvimento neuropsicomotor, tendo impacto no desenvolvimento da linguagem (Carvalho, Lemos, \& Goulart, 2016).

Nesses termos, considera-se necessária a realização de pesquisas que possam compreender as particularidades em relação ao impacto do nível socioeconômico e aspectos familiares no desenvolvimento linguístico na região Norte do Brasil, especialmente a figura paterna. Assim, o presente estudo teve como objetivo avaliar a associação do abandono paterno e fatores socioeconômicos sobre o desenvolvimento da linguagem de crianças em uma creche pública no município de Marabá (PA).

\section{Metodologia}

Trata-se de um estudo de caráter transversal e descritivo-exploratório, aprovado pelo Comitê de Ética e Pesquisa (Parecer $n^{\circ}$ 3.278.934). Foram analisadas 62 crianças, com idade entre 3 a 6 anos, e um responsável de cada uma. A coleta de dados ocorreu durante os meses de abril a dezembro de 2019, no Núcleo de Educação Infantil (NEI) Maria da Conceição Silva Pereira, localizado no município de Marabá, Pará. NEI’s são instituições públicas que fornecem educação básica para crianças de 3 a 6 anos.

Foram inclusas as crianças regularmente matriculadas e cujos pais e/ou responsáveis assinaram o Termo de Consentimento Livre e Esclarecido (TCLE). E, excluídas as que apresentaram diagnósticos ou suspeitas de quaisquer tipos de distúrbios auditivos e/ou visuais, alterações na expressão da fala, complicações nutricionais e/ou endócrinas e acometimento do sistema nervoso central.

Para a coleta de dados, os seguintes instrumentos foram utilizados:

Instrumento de Medição do Nível de Pobreza: Utilizado para coletar informações a partir dos pais e/ou responsáveis. Instrumento traduzido e adaptado no Brasil por Issler e Giugliani (1997) e composto por 13 itens: 1. Número de pessoas que comem e dormem na casa; 2. Abandono do pai/mãe; 3. Escolaridade dos pais; 4. Atividade dos pais; 5. Relação com o domicílio; 6. Tipo de casa; 7. Número de pessoas que dormem na casa versus lugares para dormir; 8. Abastecimento de água; 9. Deposição de excreta; 10. Coleta de lixo; 11. Energia elétrica; 12. Cozinha independente; 13. Eletrodomésticos do domicílio. A variação de pontuação de cada item possui uma escala de 0 a 4 pontos, cuja menor pontuação total obtida é de 7 pontos e a 
maior de 52 (Costa, Cavalcante, \& Dell'Aglio, 2015).

Teste de Triagem de Desenvolvimento de Denver II (Denver II): Possui como objetivo avaliar o desenvolvimento dos infantes desde o nascimento aos 6 anos de idade. É composto por 125 itens distribuídos nas seguintes categorias: pessoalsocial, motor fino-adaptativo, linguagem e motor grosso. Nessa pesquisa somente a categoria linguagem foi avaliada. Dentro da análise de cada habilidade foram ponderadas as seguintes possibilidades de pontuações para cada item: passou (P), falhou (F), recusou (R) ou sem oportunidade (SO). Ao final das avaliações, as crianças foram categorizadas conforme seu desempenho em normal, cautela ou atraso (Pinto et al., 2015). Após essa interpretação, o desfecho foi classificado em normal, questionável ou atraso para facilitar a análise dos dados.

Com uma semana de antecedência para o início das coletas, foi encaminhado um convite para os pais e/ou responsáveis solicitando a presença na reunião com os pesquisadores para esclarecimentos da pesquisa. Durante o primeiro contato, foi entregue aos pais e/ou responsáveis o TCLE, os que assinavam eram encaminhados para coleta de dados juntamente com seus filhos.

As avaliações ocorreram em uma sala reservada nas dependências do NEI, muito arejada e confortável. Possuía uma mesa e três cadeiras, além de fita-métrica, uma balança mecânica, piso infantil em forma de quebra-cabeças e brinquedos de acordo com a idade da criança. Cada sessão teve duração total de trinta (30) minutos e ocorreu no turno matutino e/ou vespertino, com respeito ao horário das atividades escolares. Nos primeiros quinze (15) minutos foram coletadas as informações no Instrumento de Medição do Nível de Pobreza. Nos quinze (15) minutos seguintes foi realizada a avaliação do desenvolvimento da criança através do Teste de Triagem de Desenvolvimento Denver II (Denver II).

Os dados provenientes dos instrumentos foram tabulados no programa Microsoft Excel® e analisados no software Statistical Package for Social Sciences (SPSS) versão 22.0. Foram realizadas análises estatísticas descritivas e inferenciais. A variável dependente da pesquisa foi o escore de desenvolvimento advindo do Denver II, tratada como variável dicotômica (normal ou questionável). As variáveis independentes foram obtidas pelos demais instrumentos. Visando analisar a associação entre o desfecho, se normal ou questionável na linguagem e as variáveis independentes, foram utilizados os seguintes testes: Teste de Qui-quadrado e Teste G, e para comparar as médias dos escores do Denver II foi utilizado o Teste U (Teste não paramétrico de Mann Whitney), para todos os testes adotou-se nível de significância de 5\% ( $p$ valor $<0,05$ ).

\section{Resultados}

Em nosso estudo, dentre as 62 crianças analisadas 32 (51.62\%) pertenciam ao sexo feminino e 30 (48.38\%) ao masculino, com média de idade de 61.60 meses. Em relação ao Teste de Triagem de Desenvolvimento de Denver II, 34 (54.83\%) apresentaram desenvolvimento da linguagem normal, 15 (24.20\%) com atraso e 13 (20.97\%) com resultado cautela. Visando facilitar a compreensão das análises de dados, as crianças com atraso e com cautela 28 (45.17\%), foram agrupadas em um único grupo nomeado questionável (Figura 1). 
Figura 1. Porcentagem referente ao desfecho do desenvolvimento linguístico segundo o Teste de Triagem de Denver II.

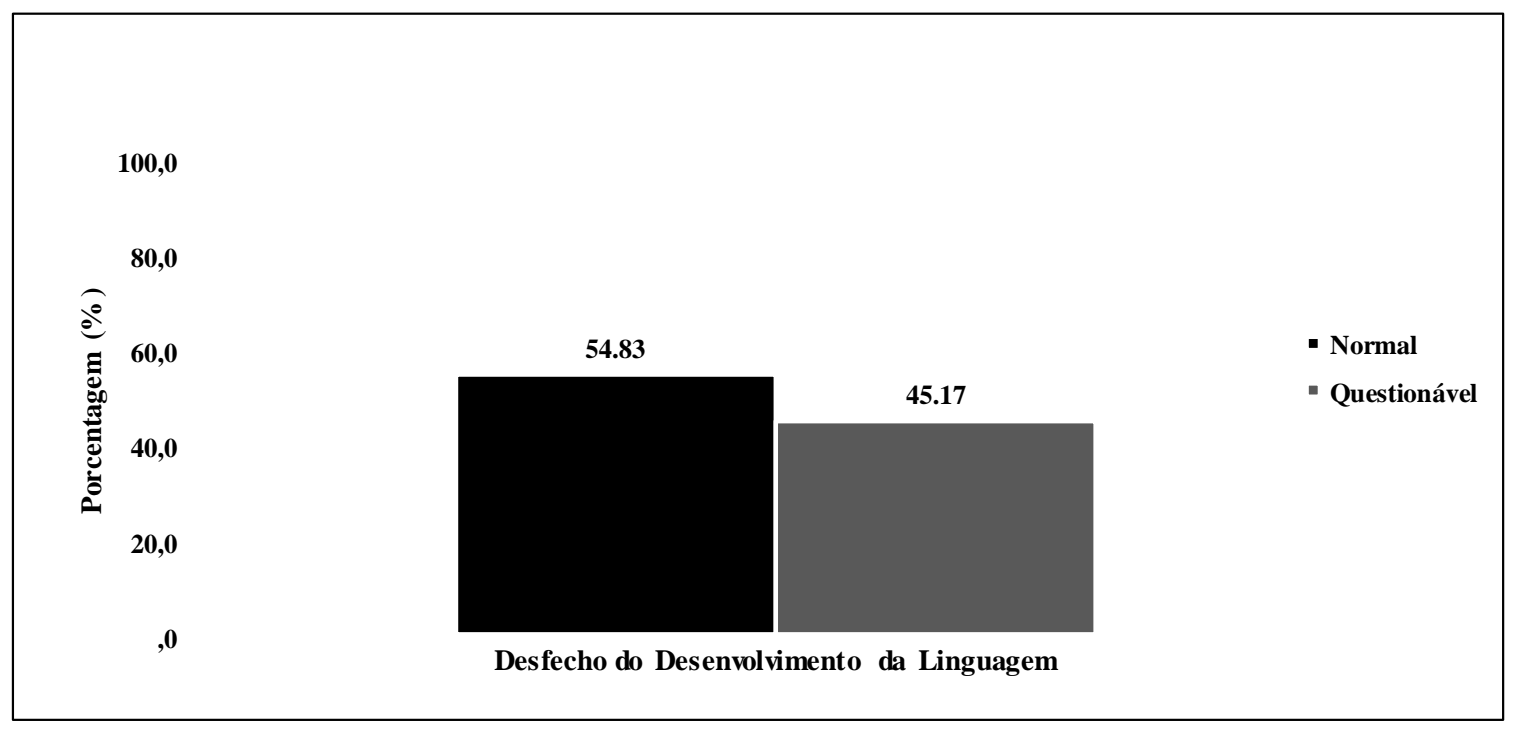

Fonte: Autores (2021).

Relativo à associação das variáveis socioeconômicas com o desfecho do Denver II na área da linguagem, verificou-se que $55(88.70 \%)$ das crianças apresentavam renda baixa superior, 4 (6.45\%) renda baixa inferior e 3 (4.80\%) situação de miséria $(\chi 2=0.925, p=0.63)$. Quanto a escolaridade do cuidador, $43(69.40 \%)$ haviam cursado até a oitava série do ensino fundamental ou apresentavam ensino médio completo/incompleto, 14 (22.60\%) da quinta a sétima série, 3 (4.80\%) até a quarta série e $2(3.20 \%)$ eram analfabetos $(\mathrm{G}=39.3, p=0.98)$. Já em relação a atividade profissional do pai, aquelas crianças cujos pais trabalhavam por tarefa, 25 (89.30\%), apresentaram desfecho questionável, enquanto aquelas que possuíam pais com trabalho regular, $16(47.10 \%)$, apresentaram desfecho normal $(\chi 2=15.56, p=0.001)$ (Tabela 1$)$.

Tabela 1. Frequência e relação das variáveis socioeconômicas com o desfecho do Teste de Triagem de Desenvolvimento de Denver II.

\begin{tabular}{|c|c|c|c|c|c|}
\hline Variável & $\begin{array}{l}\text { Questionável } \\
\text { N=28 (\%) }\end{array}$ & $\begin{array}{c}\text { Normal } \\
\mathrm{N}=34(\%)\end{array}$ & $\begin{array}{c}\text { Total } \\
\mathrm{N}=62(\%)\end{array}$ & Teste & $p$ valor \\
\hline Nível de Pobreza & & & & $0.925^{\mathrm{b}}$ & 0.63 \\
\hline Miséria & $1(3.60)$ & $2(5.90)$ & $3(4.80)$ & & \\
\hline Baixa inferior & $1(3.60)$ & $3(8.80)$ & $4(6.50)$ & & \\
\hline Baixa superior & $26(92.90)$ & $29(85.30)$ & $55(88.70)$ & & \\
\hline Escolaridade do Cuidador & & & & $39.3^{\mathrm{a}}$ & 0.98 \\
\hline Analfabeto & $1(3.60)$ & $1(2.90)$ & $2(3.20)$ & & \\
\hline Até a $4^{\mathrm{a}}$ série & $0(0.00)$ & $3(8.80)$ & $3(4.80)$ & & \\
\hline $5^{\mathrm{a}}$ a $7^{\mathrm{a}}$ serie & $7(25.00)$ & $7(20.60)$ & $14(22.60)$ & & \\
\hline $8^{\mathrm{a}} \mathrm{ou}+$ & $20(71.40)$ & $23(67.60)$ & $43(69.40)$ & & \\
\hline Atividade Profissional dos Pais & & & & $15.56^{\mathrm{b}}$ & $0.001 *$ \\
\hline Aposentado & $0(0.00)$ & $1(2.90)$ & $1(1.60)$ & & \\
\hline Trabalho por tarefa & $25(89.30)$ & $14(41.20)$ & $39(62.90)$ & & \\
\hline Trabalho regular & $3(10.70)$ & $16(47.10)$ & $19(30.60)$ & & \\
\hline Possui pequeno comércio & $0(0.00)$ & $3(8.80)$ & $3(4.80)$ & & \\
\hline
\end{tabular}

aTeste G; Teste de Qui-quadrado; * $p<0.01$

Fonte: Autores (2021). 
Já a Tabela 2 demonstra a associação entre o abandono do pai e o desfecho do Denver II. Observou-se que aquelas crianças que foram abandonadas totalmente pelo pai, 11 (39.30\%) apresentaram resultado questionável na linguagem, enquanto aquelas que tinham o pai presente, 25 (73.50\%) apresentaram desenvolvimento linguístico normal $(\mathrm{G}=8.98, p=$ $0.009)$.

Tabela 2. Frequência e relação da variável biossociodemográfica abandono do pai com o desfecho do Teste de Triagem de Desenvolvimento de Denver II.

\begin{tabular}{|c|c|c|c|c|c|}
\hline Variável & $\begin{array}{c}\text { Questionável } \\
\mathrm{N}=\mathbf{2 8}(\%)\end{array}$ & $\begin{array}{c}\text { Normal } \\
\mathrm{N}=\mathbf{3 4}(\%)\end{array}$ & $\begin{array}{c}\text { Total } \\
\mathrm{N}=62(\%)\end{array}$ & Teste & $p$ valor \\
\hline Abandono do Pai & & & & $8.98^{\mathrm{a}}$ & $0.009 *$ \\
\hline Abandono total & $11(39.30)$ & $8(23.50)$ & $19(30.60)$ & & \\
\hline Abandono parcial & $2(7.10)$ & $1(2.90)$ & $3(4.80)$ & & \\
\hline Sem abandono & $15 \quad(53.60)$ & $25(73.50)$ & $40 \quad(64.50)$ & & \\
\hline
\end{tabular}

aTeste $\mathrm{G} ; * p<0.01$.

Fonte: Autores.

\section{Discussão}

Este estudo mostrou associação do abandono paterno e dos fatores socioeconômicos sobre o desenvolvimento da linguagem em crianças de uma creche pública no município de Marabá (PA). Verificou-se que $45.17 \%$ das crianças avaliadas tiveram o desfecho de linguagem como questionável. Esse dado é similar aos valores encontrados em outros estudos realizados em creches públicas no Brasil, como o de Araújo, Quadros, Murata e Israel (2019) em que das 85 crianças de uma creche no município de Matinhos - PR, avaliadas pelo Teste de Denver II, 58.89\% tiveram o desempenho questionável na área da linguagem. No estudo de Costa, Cavalcante e D’Aglio, realizado em Unidade de Educação Infantil no município de Belém PA, também houve prevalência de crianças com suspeitas de atraso no desenvolvimento linguístico, com 59.20\% do total da amostra.

Nesse sentido, é sabido que as intervenções educacionais inseridas nos primeiros anos de vida mostram que a educação pré-escolar pode intervir positivamente nas habilidades cognitivas e sociais de uma criança, bem como no neurodesenvolvimento e na saúde. Em um estudo de coorte realizado em Pelotas foi verificado que o impacto dos centros educacionais demonstrou efeitos de curto a longo prazo no desenvolvimento cognitivo, socioemocional, escolar e até mesmo na criminalidade (Leão et al., 2021). Contudo, pesquisadores encontraram uma importante parcela de crianças com suspeita de atraso no desenvolvimento linguístico, apesar de todas estarem regularmente matriculadas no Núcleo de Educação Infantil, sugerindo forte impacto do ambiente familiar e demográfico no desenvolvimento, haja vista que foram encontrados dados significativos no que se refere à atividade profissional dos pais e ao abandono paterno (Brownell et al., 2016).

É notório que, para o adequado desenvolvimento infantil, exige-se um contexto socioeconômico propício e protetor à criança, ao contrário, a situação de vulnerabilidade social pode desencadear alterações na aquisição e no desenvolvimento da linguagem, gerando diversas consequências futuras, desde um mau desempenho escolar até dificuldades de socialização e vulnerabilidades sociais (Carvalho, Lemos, \& Goulart, 2016). Em nosso estudo uma pequena parcela das crianças encontravase em situação de extrema pobreza ou de miséria, justificando o fato da maioria dos pais possuírem nível de escolaridade equivalente ou superior ao ensino fundamental completo. Quanto maior a escolaridade materna ou paterna, maiores serão as 
probabilidades de alta renda familiar, melhores empregos, prestígio social e melhor qualidade e quantidade das habilidades parentais adquiridas para estimular o desenvolvimento infantil (Kadosaki, Luz, Macias, \& Dias, 2020).

No que tange ao nível de escolaridade do cuidador no presente estudo, este fator não demonstrou interferência de forma isolada na aquisição da linguagem. Porém, ao se considerar o alto percentual equivalente ao grupo questionável no Teste de Denver II, não se pode contraditar que outras variáveis sociodemográficas que permeiam o ambiente familiar estejam interferindo no processo do desenvolvimento de linguagem das crianças. A pesquisa de Silva, Ferreira e Queiroga (2016), aponta correlação significativa entre a escolaridade materna e o hábito de leitura, mostrando a influência nas práticas de letramento e no estímulo de aquisição da fala de seus filhos. Não foram encontrados estudos quanto ao hábito de leitura da figura paterna e o desenvolvimento linguístico infantil.

O tipo de ocupação dos pais apresentou relevância em nossos resultados, haja vista que dentre as crianças analisadas, as que possuíam pais com trabalho informal, aproximadamente $90.0 \%$, apresentaram desfecho linguístico questionável. A irregularidade do trabalho dos pais aumenta a probabilidade de instabilidade econômica e, consequentemente, gera um ambiente socioeconômico desfavorável que pode influenciar negativamente no desenvolvimento linguístico infantil. Assim, os indicadores de gradientes socioeconômicos, como ocupação laboral ou escolaridade, não determinam simplesmente a renda familiar, como também sugerem o perfil de estimulação psicossocial, o ambiente ecológico e o nível de interação parental com os infantes (González et al., 2018). Pais com o status socioeconômico mais baixo tendem a conversar menos com os seus filhos e usam um vocabulário menos diverso, além de possuírem pouco hábito de leitura e de interação por meio de brincadeiras (Brancalioni, Zauza, Karlinski, Quitaiski, \& Thomaz, 2018).

Quanto a variável abandono paterno em nosso estudo, houve correlação significativa com o atraso linguístico das crianças avaliadas. No estudo de Araújo, Mélo e Israel (2017) realizado no Município de Matinhos - PR, 57.50\% das crianças com desenvolvimento questionável para linguagem quando sujeitas ao abandono paterno apresentavam aumento em até 34 vezes na probabilidade de evoluírem com atrasos no desenvolvimento.

Fatores de riscos familiares, incluindo o abandono do pai, podem causar alterações epigenéticas e gerar estresse oxidativo, afetando fortemente o desenvolvimento neural, logo, acometendo áreas responsáveis pela associação e pela propagação da fala. De modo que, durante o desenvolvimento infantil, fatores prejudiciais, sejam eles extrínsecos ou intrínsecos, podem alterar as conexões e as funções neuronais superiores de maneira deletéria (Berens et al., 2019). Esse processo de remodelamento cerebral é caracterizado como neuroplasticidade, fenômeno com períodos críticos, os quais devem ser compreendidos e anteferidos na intervenção precoce e estão intimamente relacionados com o potencial de recuperação funcional.

Além disso, compreende-se que no período embrionário a formação de neurônios e células da glia e todos os seus processos biológicos são momentos cruciais e que após o nascimento, durante a infância, a formação de dendritos, das sinapses e do processo de mielinização e poda sináptica são os eventos mais importantes, formando até o dobro de sinapses evidenciadas nos adultos. Esse período crítico é de extrema importância, e fatores como o abandono paterno possuem alta relevância, uma vez que sua ausência não permeia apenas o seu papel paterno, mas como toda a figura social que foi construída, o apoio financeiro e os estímulos lexicais imprescindíveis na primeira infância (Malmberg et al., 2015).

Nesse ínterim, a presença do pai na infância pode ser associada a um melhor estímulo para determinadas áreas, incluindo a linguagem, uma vez que precisa de influências externas para ser adquirida. O contexto familiar da criança é um dos meios principais para aprimorar o seu desenvolvimento, principalmente no que se refere à interação filho-progenitor, pois, apesar da escola ser um instrumento essencial, a criança vive a maior parte do seu tempo no ambiente familiar, consequentemente, deve ser o lugar que tem maiores interações e estímulos (Brownell et al., 2016). 


\section{Considerações Finais}

No presente estudo foi possível verificar a influência direta de variáveis sociais como abandono paterno e fatores socioeconômicos sobre o desenvolvimento da linguagem infantil, pois as crianças que foram abandonadas totalmente pelo pai apresentaram desfecho questionável em seu desenvolvimento linguístico, juntamente com as crianças cujos pais trabalhavam por tarefa e não possuíam emprego fixo. Assim, é visível que o desenvolvimento infantil é permeado por diversos fatores e dependente de estímulos positivos para ocorrer da forma adequada, quando estes não são ofertados de forma correta, principalmente durante a primeira infância, a criança se torna mais suscetível a desenvolver atrasos, os quais se não revertidos impactarão de forma negativa todas as áreas de sua vida.

Dessa forma, políticas públicas direcionadas para o período da primeira infância são necessárias, como a criação e distribuição de cartilhas acerca do desenvolvimento infantil e a sensibilização da população através da divulgação de materiais e programas televisivos com a temática de como fornecer e quais são os estímulos necessários para que a criança se desenvolva adequadamente. Ainda, é necessário facilitar o acesso aos Núcleos de Educação Infantil para a população menos abastada financeiramente, como também realizar o investimento apropriado para o seu funcionamento através da oferta de treinamentos aos gestores e professores.

Além disso, compete ao Estado garantir aos cidadãos em situação de pobreza e miséria programas que fornecem subsídios financeiros, como também fornecer maiores taxas de vínculo empregatício, visando minimizar os diversos danos gerados por eles sobre o desenvolvimento da criança. Por fim, são necessárias mais pesquisas direcionadas a essa temática, visando aumentar as taxas de triagem para atrasos no desenvolvimento e o encaminhamento ao diagnóstico e tratamento necessários.

\section{Referências}

Aarnoudse-Moens, C. S., Duivenvoorden, H. J., Weisglas-Kuperus, N., Van Goudoever, J. B., \& Oosterlaan, J. (2013). Neonatal and parental predictors of executive function in very preterm children. Acta Paediatrica, 102(3), 282-286. https://doi.org/10.1111/apa.12101

Anme, T., Tanaka, E., Shinohara, R., Sugisawa, Y., Watanabe, T., Tomisaki, E., \& Segal, U. A (2012). Center-based child extended care: Implications for young children's development in a five-year follow-up. Sociology Mind, 2(4), 435-440. http://dx.doi.org/10.4236/sm.2012.24056

Araújo, L. B., Melo, T. R., \& Israel, V. L. (2017). Low birth weight, family income and paternal absence as risk factors in neuropsychomotor development. Journal Human Growth Development, 27(3), 272-280. https://doi.org/10.7322/jhgd.124072

Araújo, L. B., Quadros, D. A., Murata, M. P. F., \& Israel, V. L. (2019). Avaliação do desenvolvimento neuropsicomotor de crianças de 0 a 5 anos em centros de educação pública infantil. Revista CEFAC, 21(3), 1-9. http://dx.doi.org/10.1590/1982-0216/201921312918

Berens, A. E., Kumar, S., Tofail, F., Jensen, S. K., Alam, M., Haque, R., \& Nelson, C. A. (2019). Cumulative psychosocial risk and early child development: validation and use of the Childhood Psychosocial Adversity Scale in global health research. Pediatric research,86(6), 766-775. https://doi.org/10.1038/s41390-019-0431-7

Brancalioni, A. R., Zauza, A., Karlinski, C. D., Quitaiski, L. F., \& Thomaz, F. O. (2018). Desempenho do vocabulário expressivo de pré-escolares de 4 a 5 anos da rede pública e particular de ensino. Audiology Communication Research, 23, 1-9. https://doi.org/10.1590/2317-6431-2016-1836

Brownell, M. D., Ekuma, O., Nickel, N. C., Chartier, M., Koseva, I., \& Santos, R. G. (2016). A population-based analysis of factors that predict early language and cognitive development. Early Childhood. Research Quarterly, 35, 6-18. https://doi.org/10.1016/j.ecresq.2015.10.004

Carvalho, A. D., Lemos, S. M., \& Goulart, L. M. (2016). Desenvolvimento da linguagem e sua relação com comportamento social, ambientes familiar e escolar: revisão sistemática. CoDAS, 28(4), 470-479. http://dx.doi.org/10.1590/2317-1782/20162015193. PMid:27652929

Catale, C., Willems, S., Lejeune, C., \& Meulemans, T. (2012). Parental educational level influence on memory and executive performance in children. Revue Européenne de Psychologie Appliquée, 62(3), 161-171. https://doi.org/10.1016/j.erap.2012.04.003

Costa, E. F., Calvacante, L. I., \& Dell'Aglio, D. D. (2015). Perfil do desenvolvimento da linguagem de crianças no município de Belém, segundo o Teste de Triagem de Denver II. Revista CEFAC, 17(4), 1090-1102. https://doi.org/10.1590/1982-0216201517418514

Filippetti, V. (2011). Funciones ejecutivas en niños escolarizados: efectos de la edad y del estrato socioeconómico. Avances em Psicología Latinoamericana, 29(1), 98-113.

Flores, M., García-Gómez, P., \& Zunzunegui, M. V. (2014). Crisis económica, pobreza e infancia. ¿ Qué podemos esperar en el corto y largo plazo para los "niños y niñas de la crisis"? Informe SESPAS 2014. Gaceta sanitaria, 28, 132-136. https://doi.org/10.1016/j.gaceta.2014.02.021 
González, L., Cortés-Sancho, R., Murcia, M., Ballester, F., Rebagliato, M., \& Rodríguez-Bernal, C. L. (2020). The role of parental social class, education and unemployment on child cognitive development. Gaceta sanitaria, 34(1), 51-60. https://doi.org/10.1016/j.gaceta.2018.07.014

Issler, R. M., \& Giugliani, E. R. (1997). Identificação de grupos mais vulneráveis à desnutrição infantil pela medição do nível de pobreza. Jornal de Pediatria, 73(2), 101-105.

Jacobsen, G. M., Moraes, A. L., Wagner, F., \& Trentini, C. M. (2013). Qual é a participação de fatores socioeconômicos na inteligência de crianças? Revista Neuropsicologia Latinoamericana, 5(4), 32-39. http://dx.doi.org/10.5579/rnl.2013.0165

Kadosaki, D. J., da Luz, P. N. V., Macias, L. L., \& Dias, S. M. (2020). Perfil econômico e familiar de crianças com atraso no desenvolvimento neuropsicomotor em um hospital na Amazônia. Pará Research Medical Journal, 4, 0-0. https://dx.doi.org/10.4322/prmj.2019.043

Leão, O. A. D. A., Mielke, G. I., Silveira, M. F. D., Domingues, M. R., Murray, J., Neumann, N. A., \& Bertoldi, A. D. (2021). Influence of center-based child care on development of two-year-olds in a Brazilian cohort. Revista de Saúde Pública, 55(32). https://doi.org/10.11606/s1518-8787.2021055002958

Lima, S. S., Cavalcante, L. I., \& Costa, E. F. (2016). Triagem do desenvolvimento neuropsicomotor de crianças brasileiras: uma revisão sistemática da literatura. Fisioterapia e Pesquisa, 23(3), 336-342. https://doi.org/10.1590/1809- 2950/15703523032016

Malmberg, L.E., Lewis, S., West, A., Murray, E., Sylva, K., \& Stein, A. (2015). The influence of mothers' and fathers' sensitivity in the first year of life on children's cognitive outcomes at 18 and 36 months. Child Care Health Dev, 42(1),1-7. http://gx.org/0.1111/cch.12294

Manfroi E. C., Macarini S. M., \& Vieira M. L (2011). Comportamento parental e o papel do pai no desenvolvimento infantil. Journal of Human Growth and Development, 21(1), 59-69.

Mélo, T. R., Lucchesi, V. O., Junior, E. J., \& Signorelli, M. C. (2020). Caracterização do desenvolvimento neuropsicomotor e de linguagem de crianças atendidas por grupos no Núcleo Ampliado de Saúde da Família: Uma abordagem interprofissional. Revista CEFAC, 22(3), 1-10. http://dx.doi.org/10.1590/1982-0216/202022314919

Morais, R. L., Carvalho, A. M., \& Magalhães, L. C. (2016). O contexto ambiental e o desenvolvimento na primeira infância: estudos brasileiros. Journal of Physical Education, 27(1), 2-14. https://doi.org/10.4025/jphyseduc.v27i1.2714

Pinto, F. C., Isotani, S. M., Sabatés, A. L., \& Perissinoto, J. (2015). Denver II: Comportamentos propostos comparados aos de crianças paulistanas. Revista CEFAC, 17(4), 1262-1269. https://doi.org/10.1590/1982-0216201517418214

Richter, L. M., Daelmans, B., Lombardi, J., Heymann, J., Boo, F. L., Behrman, J. R., \& Dua, T. (2017). Investing in the foundation of sustainable development: pathways to scale up for early childhood development. The Lancet, 389(10064), 103-118. https://doi.org/10.1016/S0140-6736(16)31698-1

Silva, A. C. F. D., Ferreira, A. A., \& Queiroga, B. A. M. D. (2014). Desenvolvimento da narrativa oral e o nível de escolaridade materna. Revista CEFAC, 16(1), 174-186. http://dx.doi.org/10.1590/1982-021620146412

Tran, T. D., Luchters, S., \& Fisher, J. (2017). Early childhood development: impact of national human development, family poverty, parenting practices and access to early childhood education. Child: care, health and development, 43(3), 415-426. https://doi.org/10.1111/cch.12395

Trapp, E. H., \&Andrade, R. D. (2017). As consequências da ausência paterna na vida emocional dos filhos. Revista Ciência Contemporânea, 2 (1), 45-53.

Zago, J. T., Pinto, P. A., Leite, H. R., Santos, J. N., \& Morais, R. L. (2017). Associação entre o desenvolvimento neuropsicomotor e fatores de risco biológico e ambientais em crianças na primeira infância. Revista Cefac, 19(3), 320-329. https://doi.org/10.1590/1982-0216201719314416 